\title{
Anterograde and retrograde amnesia following bitemporal infarction
}

\author{
A. Schnider, M. Regard and T. Landis \\ Department of Neurology, University Hospital, Zürich, Switzerland \\ Correspondence to: Dr med. A. Schnider, Abt. für Neuropsychologische Rehabilitation, \\ Inselspital, $\mathrm{CH}-3010$ Bern, Switzerland
}

\begin{abstract}
A patient suffered very severe anterograde and retrograde amnesia following infarction of both medial temporal lobes (hippocampus and adjacent cortex) and the left inferior temporo-occipital area. The temporal stem and the amygdala were intact; these structures do not appear to be critical for new learning in humans. Extension of the left-sided infarct into the inferior temporo-occipital lobe, an area critically involved in visual processing, appears to be responsible for our patient's loss of remote memories.
\end{abstract}

Keywords: Amnesia - Amygdala - Hippocampus - Inferotemporal cortex - Medial temporal lobe

\section{INTRODUCTION}

Impaired learning of new information (anterograde amnesia) may result from damage to diencephalic structures, the basal forebrain, or medial temporal lobes (Signoret, 1985; Von Cramon et al., 1990; Squire and Zola-Morgan, 1991). It is still controversial what medial temporal structures contribute to learning. Hippocampal damage alone may cause significant, albeit incomplete anterograde amnesia (Zola-Morgan et al., 1986; Victor and Agamanolis, 1990). Very severe amnesia has been reported only after large lesions involving both the hippocampus and adjacent structures (Scoville and Milner, 1957; Cermak and O'Connor, 1983; Damasio et al., 1985). It is unclear whether this is due to additional damage of the amygdala, parahippocampal cortex, or both. Extensive loss of old memories (retrograde amnesia) has been attributed to damage of the temporal lobes (Cermak and O'Connor, 1983; Kapur et al., 1992), the basal forebrain (Damasio et al., 1985; Morris et al., 1992), and the brainstem (Goldberg et al., 1981). Only one of these reports concerned a patient with a focal medial forebrain lesion (Morris et al., 1992); the others had widespread cerebral damage after trauma or encephalitis. We present a patient with very severe anterograde and retrograde amnesia following bilateral medial temporal and left inferior temporo-occipital infarction. Lesion analysis allowed us to further delineate the memory-critical structures in the medial temporal lobe.

\section{CASE REPORT}

A 66 year old, right-handed retired mechanic had a history of myocardial infarction and aortocoronary bypass 6 years previously. He recovered well and fully resumed work till his retirement 1 year ago. $\mathrm{He}$ was in excellent condition and was especially respected by family members for his good memory and his ability to orient himself in new locations.

On the morning of admission, he was confused on awakening and could not orient himself in his bedroom. Physical examination revealed a right homonymous upper quadrantanopia and a discrete right hemiparesis that resolved within 4 days. Doppler ultrasound examination showed non-stenotic plaques in the carotid bifurcation on both sides, while the vertebral and basilar arteries were normal. An echocardiogram evidenced an anterior myocardial aneurysm with mural thrombi, which prompted anticoagulation therapy. Magnetic resonance imaging (MRI) on the seventh day revealed infarction of both medial temporal lobes and the left inferior temporo-occipital area (Fig. 1). Initial confusion cleared within a few days but the patient remained severely disoriented in time and space and could give no account of his personal history. The only people he recognized were his wife and his children. When he briefly left his hospital bedroom, he could not find his way back.

Neuropsychological testing was performed between 2 and 4 weeks after the stroke and at 3 and 6 months 

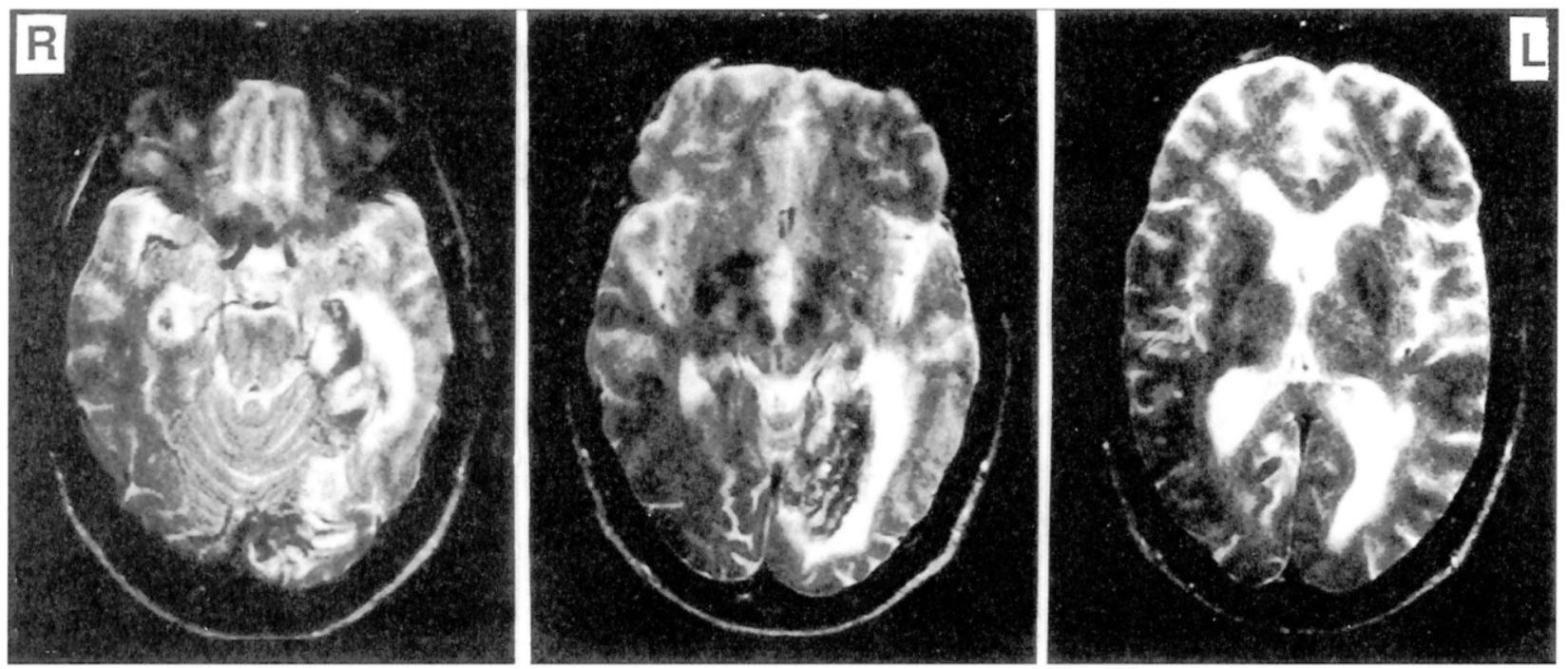

FIG. 1. MRI (TR 2000, TE 100, gadolinium enhanced) horizontal sections, showing bilateral medial temporal lobe infarcts. The lesion on the left side is much larger and extends into the inferior occipital lobe. [Reprinted from Schnider et al., (1992) with permission, (C) 1992 American Medical Association].

TABLE I. Anterograde memory tests performed 2-4 weeks after stroke. Performance in these tests at follow-up 3 and 6 months post-stroke was similar except for slightly improved digit span (6 forward, 4 backward)

\begin{tabular}{lc}
\hline Digit span forward & 5 \\
$\quad$ backward & 3 \\
Corsi cubes forward & 5 \\
$\quad$ backward & 5 \\
Rey verbal learning test (15 words; Rey, \\
1964 ) \\
Trial 1 & 2 \\
Trial 5 & 4 \\
Recall (5 min) & 0 \\
Recognition (5 min) & 0 \\
Rey visual design learning test & \\
abbreviated (10 designs; Rey, 1964) & \\
Trial 1 & 0 \\
Trial 4 & 1 \\
Recall (5 min) & 0 \\
Recognition (5 min) & 0 \\
Pantomime imitation (10 meaningful & \\
pantom.) & \\
Immediate imitation & All correct \\
Recall 5 min & 0 \\
Rey-Osterrieth Copy (Rey, 1941) & 36 \\
Recall (5 min) & 0 \\
\hline
\end{tabular}

post-stroke. While the patient appeared to be indifferent to his memory disturbance in the first days, he started to be concerned after about 2 weeks and often complained about his bad memory. He never confabulated but stated in response to questions that he did not remember. He was friendly and alert and cooperated fully in the examinations, but he never recalled either the examiners or events of previous examinations.

His speech was fluent and correct without paraphasia. Repetition was normal. Auditory comprehension was good, even for syntactically complex sentences. Long sentences could not be repeated and multistep commands executed to the extent the sentence could be repeated. There was a severe naming disorder involving visual, tactile, olfactory and auditory nonverbal material [the naming disorder is discussed elsewhere (Schnider et al., 1992)]. Reading was fluent without paralexia but comprehension was impaired as only the very last item was recalled. Writing to dictation and calculation of two-digit numbers were flawless. There was no ideomotor apraxia to verbal command. He easily recognized and outlined visually complex material, such as illusory contours or overlapping drawings. Facial matching in the Benton-Van Allen test (Benton and Van Allen, 1968; score 41) and colour recognition were normal. Verbal fluency (Thurstone and Thurstone, 1962) and non-verbal fluency in the five-point test (Regard et al., 1982) were abnormal because the patient forgot the instructions and committed too many perseverative errors. Colourword interference (Stroop, 1935) was slightly abnormal.

Both anterograde and retrograde amnesia were extremely severe. Table I summarizes the results of anterograde memory testing. Five minutes after presentation the patient did not recall any item from a list of words (Rey, 1964), designs (Rey, 1964), and

88 Behavioural Neurology . Vol 7 . 1994 


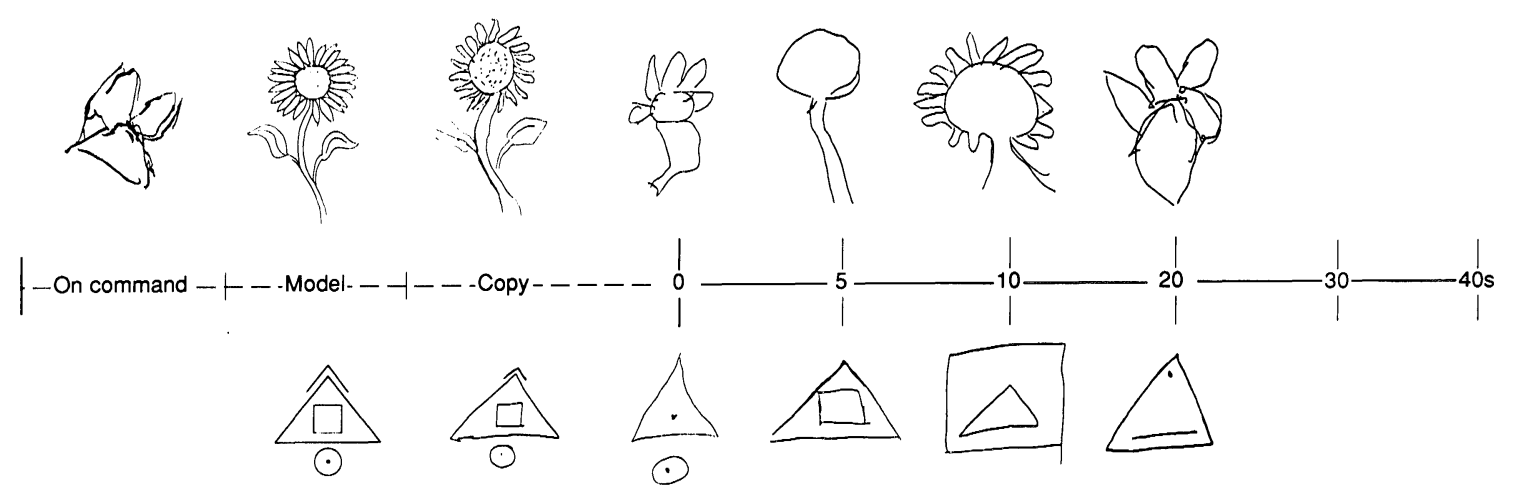

FIG. 2. Test of anterograde non-verbal memory. Above: on the left, the patient's drawing of a flower to command is shown. The patient was then asked to copy the model drawing of a flower ("copy") and then to draw the flower again from memory. This procedure of copying and then drawing from memory was repeated a total of six times with randomly chosen intervals of $0,5,10,20,30$ and $40 \mathrm{~s}$ between copying and drawing from memory. The figure shows the decline of the drawings with increasing time interval after copying the flower. After an interval of $20 \mathrm{~s}$, the drawing resembles the initial one made on command. After intervals of 30 or $40 \mathrm{~s}$, the patient was no longer aware of having drawn anything. Below: the same procedure with a geometric figure.

pantomimes and could not recall any part of the correctly copied complex figure of Rey (1941). He appeared to be unaware of having been presented with the material at all. For the recognition test the 15 words and 10 designs from the target lists were randomly intermixed with an equal number of distractors and the patient was asked to indicate for each item whether he had previously seen it (instruction: "Do you remember having been told this word/seen this figure before?'). He did not recognize any word or design. Figure 2 shows the result of a non-verbal learning test demonstrating that degradation of presented material happened almost immediately: after 30 seconds, during which interference was created by having the patient count backwards, he was no longer aware of having drawn anything.

Skill learning was intact in a task similar to mirror-drawing: the patient was requested to overdraw a square with a circle and a cross inside. Visual feedback was provided on a video screen. The video camera was attached above the drawing panel and turned in such a way that left to right movements of the hand appeared on the screen as up to down movements. In five subsequent trials, both speed (first trial, $200 \mathrm{~s}$; fifth trial, $115 \mathrm{~s}$ ) and precision increased although the patient never remembered the drawing. After $24 \mathrm{~h}$ performance was similar to the fifth trial.

Retrograde amnesia was probed through an extensive interview concerning knowledge about important events, famous people and autobiographical memory from all decades since the 1930s. Autobiographical information was checked by his wife. On no single item could he provide details, names or dates. For example, he did not know who the adversaries in the Second World War were and he could not tell the name of any past or present member of the Swiss government or the American president. He did not recognize famous people of the century (politicians, movie stars) on photographs but immediately named pictures of family members. Autobiographical knowledge was equally affected. For example, he did not know the name of his best friend at school, did not remember the date or place of his wedding and could not tell the year of birth of his children. He was unaware of his myocardial infarction and bypass operation 6 years previously. All he could provide were vague ideas of some major events (e.g. that "Chernobyl" was "a bad event"). The retrograde amnesia extended back to his childhood years and provided no clear time gradient except for the last premorbid year, for which even vague ideas were lacking.

\section{Course}

Over the next months, the amnesia persisted. The patient appeared to immediately forget any event. For example, he would complain that he had to spend all day at home $5 \mathrm{~min}$ after returning home from a day-long hike with his wife. He read the newspaper several times a day but could never tell what he had read. He had no recollection of a plane crash near his home. Retrograde amnesia remained equally dense: his long-time friends remained strangers to him (he adjusted to this by responding to the greetings of others with the same tone of voice but he regularly indicated to his wife that he did not know who he had greeted). He did not recognize his home 

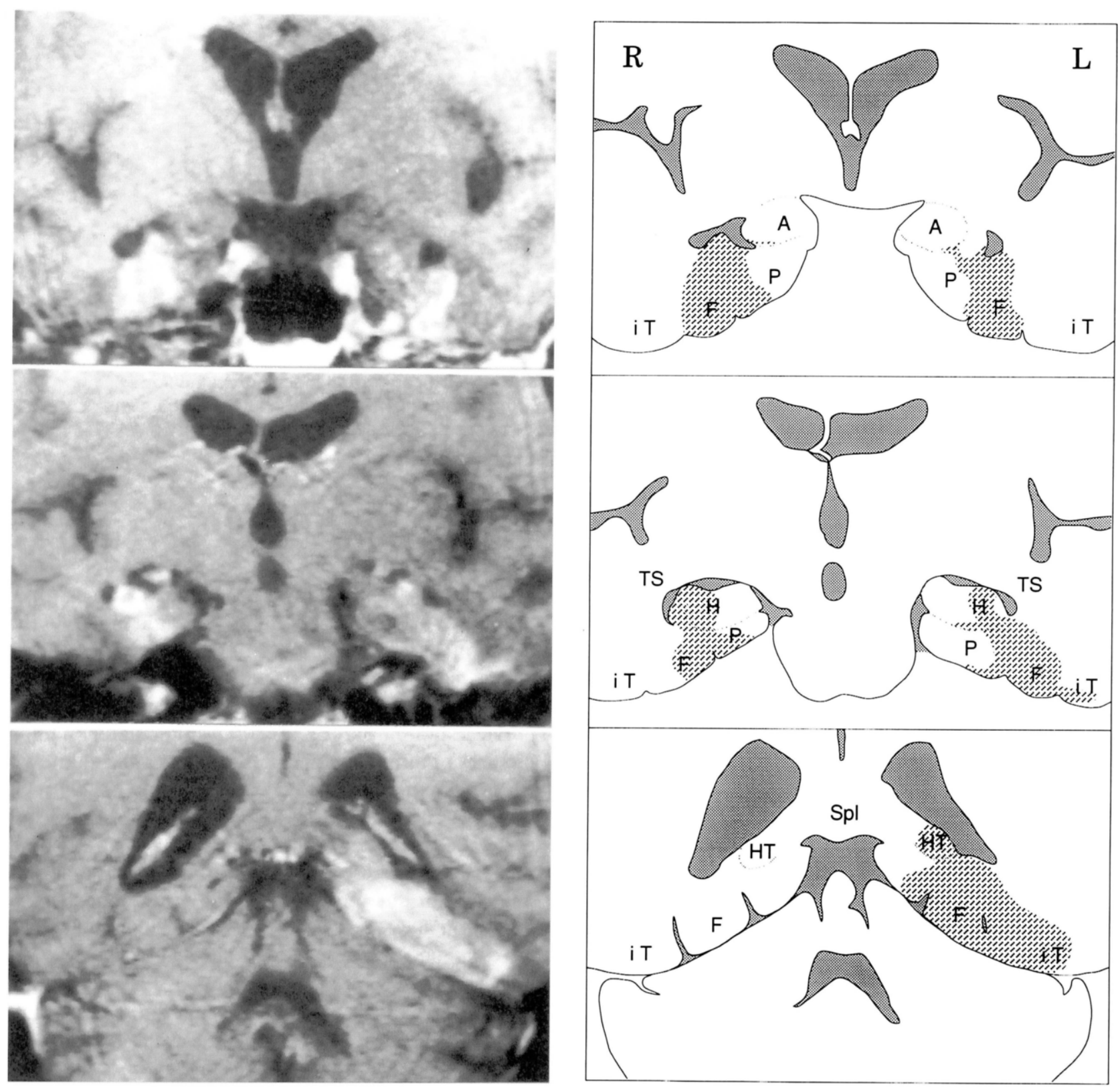

FIG. 3. MRI (TR 2000, TE 100, gadolinium enhanced), coronal sections through the medial temporal lobe (left), and outline of the lesion area based on the atlas by Duvernoy (1988) (right). A, amygdala; H, hippocampus; HT, hippocampal tail; P, parahippocampal gyrus; F, fusiform gyrus; iT, inferior temporal gyrus; TS, temporal stem; Spl, splenium of the corpus callosum.

town. The only place where he could orient himself was his own house and premises. At follow-up examinations after 3 and 6 months, digit span was slightly better ( 6 forward, 4 backward). Memory performance was similar to previous examinations (Table I).

\section{Lesion analysis}

MRI revealed an infarct involving both medial tempo- ral lobes and the left inferior temporo-occipital area (Fig. 1). On the left, the hippocampus, the parahippocampal, fusiform and posterior inferior temporal gyri as well as the inferior aspect of the occipital lobe were involved (Fig. 3). On the right, a smaller infarct involved the body of the hippocampus and the anterior half of the parahippocampal and fusiform gyri. The amygdala and the temporal stem were intact on both sides.

90 Behavioural Neurology . Vol 7 . 1994 


\section{DISCUSSION}

The role of the medial temporal lobes for learning of new information has been recognized at least since 1957, when Scoville and Milner reported the case of H.M., a patient in whom surgical removal of both medial temporal lobes including the hippocampus, the amygdala and adjacent tissue for epilepsy treatment had produced most pervasive anterograde amnesia which has since persisted (Corkin, 1984). It was unclear, however, precisely what medial temporal structure was so critical for learning. Horel (1978), primarily on the basis of animal studies, claimed that lesions of the temporal stem, a structure which had also been severed in H.M., produced amnesia. Human studies did not allow to prove this view because there has never been a report of an isolated temporal stem lesion. The role of the hippocampus, on the other hand, was demonstrated in several reports of patients with isolated hippocampal lesions following asphyxia (Zola-Morgan et al., 1986; Victor and Agamanolis, 1990). These patients had significant, albeit incomplete amnesia. Apart from H.M., very severe amnesia was only reported in cases with widespread lesions following encephalitis (Cermak anWO'Connor, 1983; Damasio et al., 1985), indicating that very severe amnesia requires damage beyond the hippocampus. Our patient is exceptional because he suffered the same degree of amnesia as H.M. following a focal brain lesion. The analysis of his lesion allows two important conclusions: (1) very severe anterograde amnesia does not require a lesion of the temporal stem (Fig. 3); (2) very severe anterograde amnesia does not require damage of the amygdala. A role of the amygdala for human learning cannot entirely be excluded, however: Tranel and Hyman (1990) described modality-specific visuospatial memory deficits in a patient with bilateral degeneration of the amygdala due to Urbach-Wiethe syndrome (however, the patient probably had complex partial seizures). Sarter and Markowitsch (1985) suggested that the amygdala may process emotional aspects of events. But Scoville and Milner's (1957) observation that bilateral amygdalectomy did not significantly affect new learning and the evidence from our case indicate that the amygdala is not essential for learning of new information. This conclusion is also consistent with recent studies on amnesia following thalamic infarction: lesions associated with amnesia appear to regularly involve the anterior thalamus with the mamillo-thalamic tract, whose major medial temporal input comes from the hippocampus. Lesions restricted to the dorso-medial nucleus, which is strongly connected with the amygdala, apparently do not produce amnesia (Von Cramon et al., 1985; Graff-Radford et al., 1990).

These conclusions also agree with animal studies: Zola-Morgan and Squire (1982), using a delayed non-matching-to-sample task in monkeys, demonstrated impaired memory after lesions involving the hippocampus-amygdala, but not the temporal stem. Further studies indicated that lesions of the hippocampus, but not of the amygdala - as suggested before by Mishkin (1982) - produced amnesia (Zola-Morgan et al., 1989). Very severe amnesia, however, required additional damage beyond the hippocampus, involving the perihippocampal, perirhinal and entorhinal cortex (Squire and Zola-Morgan, 1991). These structures are adjacent to the hippocampus and amygdala and are equivalent to those damaged in our patient (Fig. 3).

Animal experiments suggest that the hippocampus has a time-limited role for memory storage (ZolaMorgan and Squire, 1990); removal of the hippocampus does not affect information that monkeys acquired more than 8 weeks before operation. In humans, this span is considerably longer; H.M. had manifest retrograde amnesia covering 2 to 3 preoperative years but formal testing revealed deficits up to 10 years pre-operatively (Corkin, 1984). Our patient's retrograde amnesia, which had no clear time gradient and extended back to childhood years, is thus not explained by the hippocampal lesions alone. Lesions of the temporal poles (Kapur et al., 1992), the basal forebrain (Damasio et al., 1985), and the mesencephalic reticular formation (Goldberg et al., 1981) have been implicated in retrograde amnesia but all of these patients had widespread brain damage after trauma or encephalitis. Morris et al. (1992) reported a patient with severe anterograde and retrograde amnesia after removal of a low-grade astrocytoma from the right medial forebrain, supporting the view that this area is critical for memory (the patient had a 25 year history of poorly controlled complex partial seizures, however; the "retrograde amnesia" may, at least in part, have been an anterograde amnesia due to the seizures). In view of modern ideas about distributed storage of memories (Squire, 1987) it is likely that lesions of several brain areas can cause retrograde amnesia. We suggest that the left inferior temporo-occipital lesion extension was critical for our patient's extensive retrograde amnesia. The inferior temporo-occipital cortex is critical for visual processing (Fuster and Jervey, 1981; Dean, 1982) and has been considered a possible storage site for visual information (Mishkin, 1982; Squire, 1987). Retrograde amnesia following inferotemporal lesions could be based on impaired re-visualization of old 
memories-as recently suggested by O'Connor et al. (1992) and Ogden (1993) - or actual loss of old information.

\section{Acknowledgements}

We thank Professors L. R. Squire and J. M. Fuster for helpful discussion and review of a draft of the paper. Study supported by a grant from the Swiss National Foundation to Dr Schnider.

\section{REFERENCES}

Benton AL and Van Allen MW (1968) Impairment in facial recognition in patients with cerebral disease. Cortex, 4, 344-358.

Cermak LS and O'Connor M (1983) The anterograde and retrograde retrieval ability of a patient with amnesia due to encephalitis. Neuropsychologia, 21, 213-234.

Corkin S (1984) Lasting consequences of bilateral medial temporal lobectomy: clinical course and experimental findings in H.M. Seminars in Neurology, 4, 249-259.

Damasio AR, Eslinger PJ, et al. (1985) Multimodal amnesic syndrome following bilateral temporal and basal forebrain damage. Archives of Neurology, 42, 252-259.

Dean, P (1982) Visual behavior in monkeys with inferotemporal lesions. In: Analysis of Visual Behavior (Eds DJ Ingle, MA Goodale and RJW Mansfield), pp. 587-628. MIT Press, Cambridge.

Duvernoy HM (1988) The Human Hippocampus. An Atlas of Applied Anatomy. J.F. Bergmann Verlag, Munich.

Fuster JM and Jervey JP (1981) Inferotemporal neurons distinguish and retain behaviorally relevant features of visual stimuli. Science, 212, 952-955.

Goldberg E, Antin SP, et al. (1981) Retrograde amnesia: Possible role of mesencephalic reticular activation in long-term memory. Science, 213, 1392-1394.

Graff-Radford NR, Tranel D, et al. (1990) Diencephalic amnesia. Brain, 113, 1-25.

Horel JA (1978) The neuroanatomy of amnesia. A critique of the hippocampal memory hypothesis. Brain, 101, 403-445

Kapur N, Ellison D, et al. (1992) Focal retrograde amnesia following bilateral temporal lobe pathology. Brain, 115, 73-85.

Mishkin M (1978) Memory in monkeys severely impaired by combined but not by separate removal of amygdala and hippocampus. Nature, 273, 297-298.

Mishkin M (1982) A memory system in the monkey. Philosophical Transactions of the Royal Society of London, 298, 85-95.

Morris MK, Bowers DW, et al. (1992) Amnesia following a discrete basal forebrain lesion. Brain, 115, 1827-1847.

O'Connor M, Butters N, Miliotis P, Eslinger P and Cermak LS (1992) The dissociation of anterograde and retrograde amnesia in a patient with Herpes encephalitis. Journal of Clinical and Experimental Neuropsychology, 14, 159-178.

Ogden JA (1993) Visual object agnosia, prosopagnosia, achromatopsia, loss of visual imagery, and autobiographical amnesia following recovery from cortical blindness: Case M. H. Neuropsychologia, 31, 571-589.
Regard M, Strauss E and Knapp P (1982) Children's production on verbal and non-verbal fluency tasks. Perceptual and Motor Skills, 55, 839-844.

Rey A (1941) L'examen psychologique dans les cas d'encephalopathie traumatique. Archives de Psychologie, 112, 286-340.

Rey A (1964) L'examen clinique en psychologie. Presses universitaires de France, Paris.

Sarter M and Markowitsch HJ (1985) The amygdala's role in human mnemonic processing. Cortex, 21, 7-24.

Schnider A, Landis T, et al. (1992) Dissociation of color from object in amnesia. Archives of Neurology, 49, 982985.

Scoville WB and Milner B (1957) Loss of recent memory after bilateral hippocampal lesions. Journal of Neurology, Neurosurgery and Psychiatry, 20, 11-21.

Signoret JL (1985) Memory and amnesia. In: Behavioral Neurology (Ed. MM Mesulam), pp. 169-192. F. A. Davis, Philadelphia.

Squire LR (1987) Memory and Brain. Oxford University Press, New York.

Squire LR and Zola-Morgan S (1991) The medial temporal lobe memory system. Science, 253, 1380-1386.

Stroop JR (1935) Studies of interference in serial verbal reactions. Journal of Experimental Psychology, 18, 643662.

Thurstone LL and Thurstone TG (1962) Primary Mental Abilities (rev.). Science Research Associates, Chicago.

Tranel D and Hyman BT (1990) Neuropsychological correlates of bilateral amygdala damage. Archives of Neurology, 47, 349-355.

Victor M and Agamanolis D (1990) Amnesia due to lesions confined to the hippocampus: a clinical-pathologic study. Journal of Cognitive Neuroscience, 2, 246-257.

Von Cramon DY, Hebel N and Schuri U (1985) A contribution to the anatomical basis of thalamic amnesia. Brain, 108, 993-1008.

Von Cramon DY, Hebel N and Ebeling U (1990) Anatomical considerations on memory and learning deficits due to focal cerebral lesions in man. In: The Biology of Memory (Eds LR Squire and E Lindenlaub), pp. 527540. F. K. Schattauer Verlag, Stuttgart.

Zola-Morgan S and Squire LR (1982) The neuroanatomy of amnesia: amygdala-hippocampus versus temporal stem. Science, 218, 1337-1339.

Zola-Morgan S and Squire LR (1990) The primate hippocampal formation: evidence for a time-limited role in memory storage. Science, 250, 288-290.

Zola-Morgan S, Squire LR and Amaral DG (1986) Human amnesia and the medial temporal region: enduring memory impairment following a bilateral lesion limited to field CA1 of the hippocampus. Journal of Neuroscience, 6, 2950-2967.

Zola-Morgan S, Squire LR and Amaral DG (1989) Lesions of the amygdala that spare adjacent cortical regions do not impair memory or exacerbate the impairment following lesions of the hippocampal formation. Journal of Neuroscience, 9, 1922-1936.

(Received January 1994; accepted 13 May 1994) 


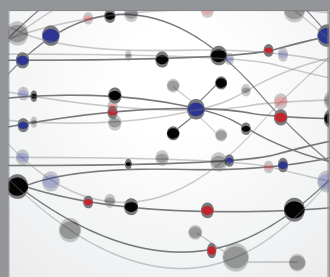

The Scientific World Journal
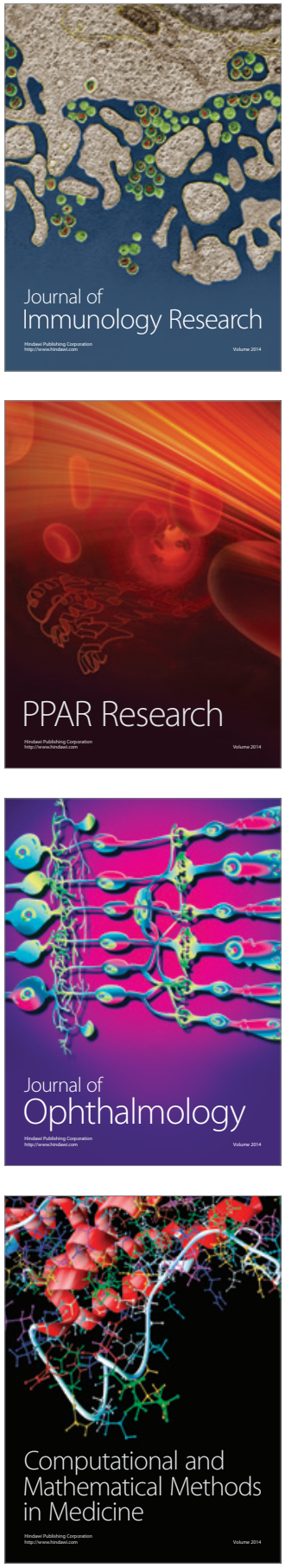

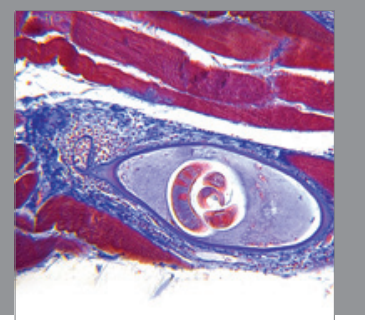

Gastroenterology

Research and Practice
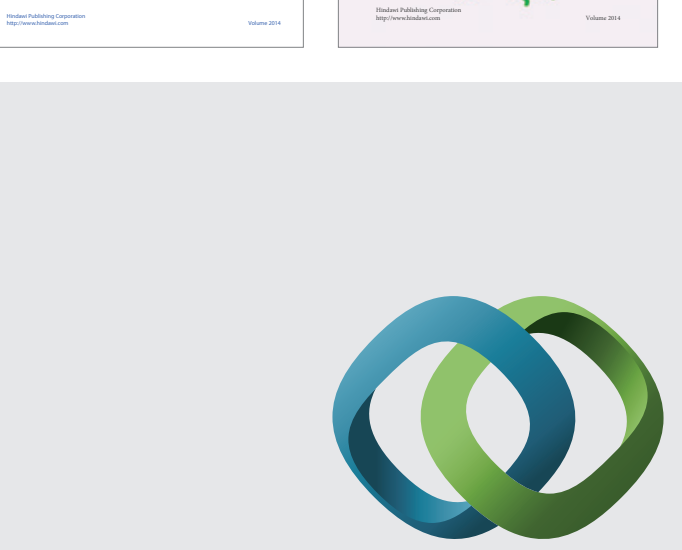

\section{Hindawi}

Submit your manuscripts at

http://www.hindawi.com
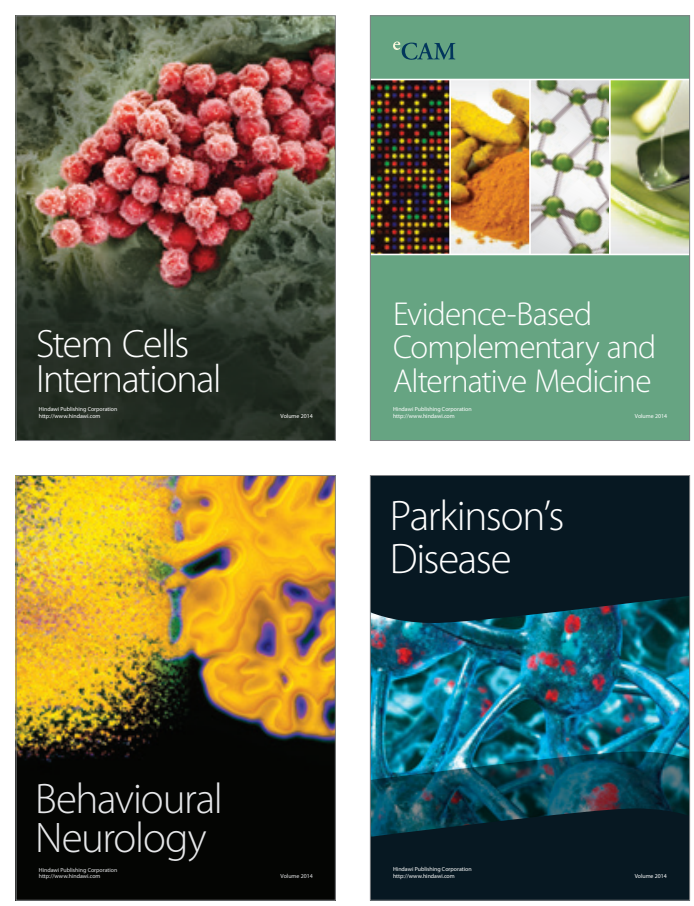

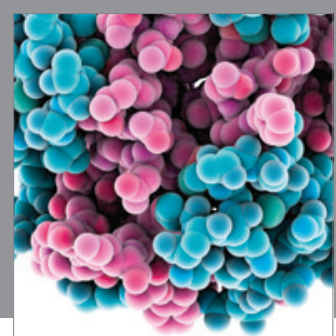

Journal of
Diabetes Research

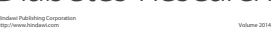

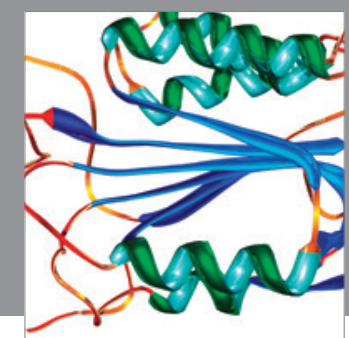

Disease Markers
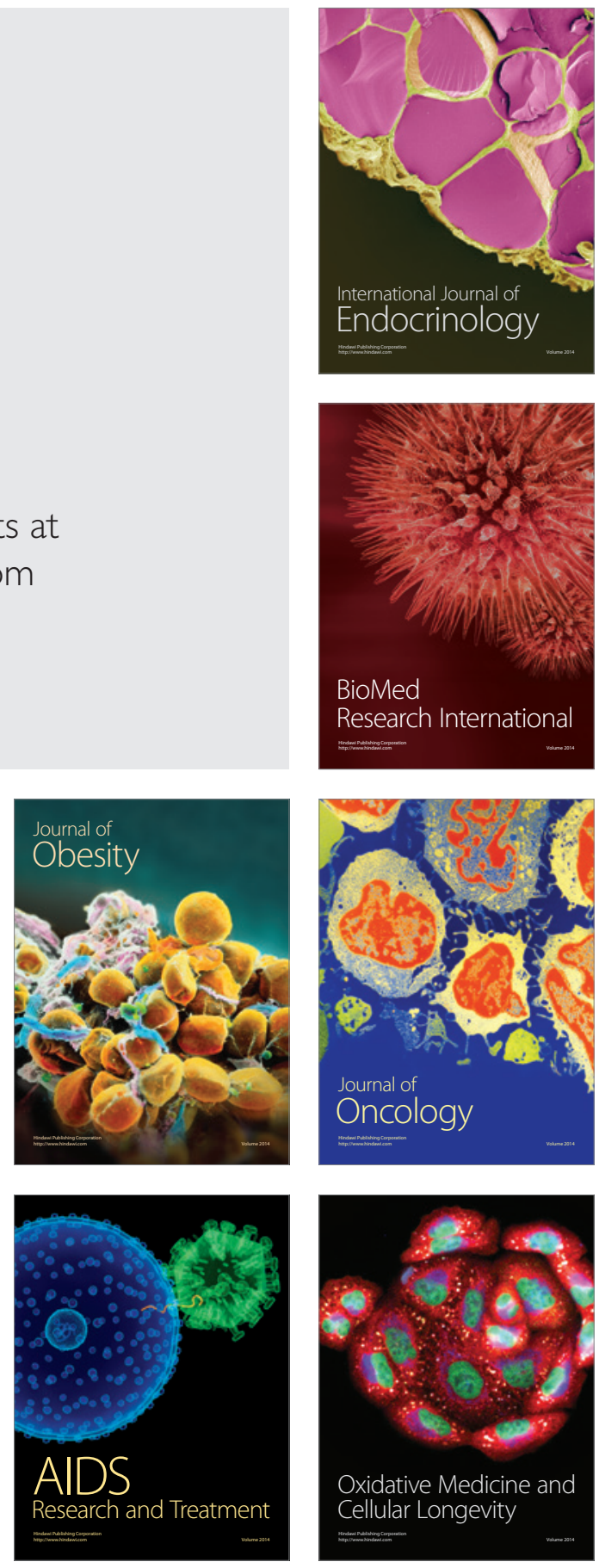